\title{
High burden of RSV hospitalization in very young children: a data linkage study
}

\author{
N. HOMAIRA ${ }^{1 *}$, J.-L. OEI ${ }^{1,2}$, K-A. MALLITT ${ }^{1}$, M. E. ABDEL-LATIF ${ }^{3}$, \\ L. HILDER ${ }^{4}$, B. BAJUK ${ }^{5}$, K. LUI ${ }^{1}$, M. FERSON ${ }^{6,7}$, A. NURKIC ${ }^{6}$, \\ G. M. CHAMBERS ${ }^{4}$, W. RAWLINSON ${ }^{8,9,10}$, T. SNELLING ${ }^{11,12,13}$ AND A. JAFFE SA $^{1,14}$ \\ ${ }^{1}$ Discipline of Paediatrics, School of Women's and Children's Health, Faculty of Medicine, UNSW Australia; \\ ${ }^{2}$ Department of Newborn Care, Royal Hospital for Women, Randwick, Sydney, Australia; ${ }^{3}$ Department of \\ Neonatology, Centenary Hospital, Australian National University; ${ }^{4}$ National Perinatal Epidemiology \& Statistics \\ Unit, Centre for Big Data Research in Health and School of Women's and Children's Health, UNSW Australia; \\ ${ }^{5}$ NSW Pregnancy and Newborn Services Network, Sydney Children's Hospitals Network, Westmead, NSW, \\ Australia; ${ }^{6}$ Public Health Unit, South Eastern Sydney Local Health District, NSW, Australia; ${ }^{7}$ School of \\ Public Health \& Community Medicine, Faculty of Medicine, UNSW Australia; ${ }^{8}$ Serology and Virology \\ Division, SEALS Microbiology, Prince of Wales Hospital, Randwick, NSW, Australia; ${ }^{9}$ School of Medical \\ Sciences, UNSW Australia; ${ }^{10}$ School of Biotechnology and Bimolecular Sciences, UNSW Australia; ${ }^{11}$ Princess \\ Margaret Hospital, Perth, Western Australia; ${ }^{12}$ Wesfarmers Centre of Vaccines \& Infectious Diseases, Telethon \\ Kids Institute, University of Western Australia; ${ }^{13}$ Menzies School of Health Research and Charles Darwin \\ University, Darwin; ${ }^{14}$ Respiratory Department, Sydney Children's Hospital, Randwick, Sydney, Australia
}

Received 28 July 2015; Final revision 28 October 2015; Accepted 4 November 2015; first published online 2 December 2015

\section{SUMMARY}

Linked administrative population data were used to estimate the burden of childhood respiratory syncytial virus (RSV) hospitalization in an Australian cohort aged $<5$ years. RSV-coded hospitalizations data were extracted for all children aged $<5$ years born in New South Wales (NSW), Australia between 2001 and 2010. Incidence was calculated as the total number of new episodes of RSV hospitalization divided by the child-years at risk. Mean cost per episode of RSV hospitalization was estimated using public hospital cost weights. The cohort comprised of 870314 children.

The population-based incidence/1000 child-years of RSV hospitalization for children aged $<5$ years was 4.9 with a rate of 25.6 in children aged $<3$ months. The incidence of RSV hospitalization (per 1000 child-years) was 11.0 for Indigenous children, 81.5 for children with bronchopulmonary dysplasia (BPD), $10 \cdot 2$ for preterm children with gestational age (GA) 32-36 weeks, $27 \cdot 0$ for children with GA 28-31 weeks, $39 \cdot 0$ for children with GA $<28$ weeks and $6 \cdot 7$ for term children with low birthweight. RSV hospitalization was associated with an average annual cost of more than AUD 9 million in NSW. RSV was associated with a substantial burden of childhood hospitalization specifically in children aged $<3$ months and in Indigenous children and children born preterm or with BPD.

Key words: Epidemiology, estimating disease prevalence, global vaccine issues, infectious disease epidemiology, paediatrics.

\section{INTRODUCTION}

Globally, acute lower respiratory tract infections (ALRIs), specifically bronchiolitis [1] are leading causes of childhood morbidity, hospitalization and death. Respiratory syncytial virus (RSV) is one of the most common respiratory viral causes for

\footnotetext{
* Author for correspondence: Dr N. Homaira, Discipline of Paediatrics, School of Women's and Children's Health, UNSW Australia, Level-3, Emergency Wing, Sydney Children's Hospital, Randwick, NSW 2031, Australia. (Email: n.homaira@unsw.edu.au)
} 
childhood ALRI [2-4]. Almost 90\% of children are infected by the virus by their second birthday $[5,6]$. Studies suggest that the rate of RSV-coded hospitalization in children aged $<5$ years ranges between 1 and $4 / 1000$ children per year with the rate highest for children aged $<6$ months $[6,7]$.

Indigenous children are at increased risk of severe respiratory illness associated with RSV [8]. Data from the USA suggest the rates of RSV hospitalization in American Indian and Alaskan native children are at least 10 times higher than children from the general population. The high rate in these children has been attributed to limited access to health facilities, lack of optimal sanitation facilities and household crowding [9].

Children born preterm [gestational age (GA) $<37$ weeks] and with chronic lung disease of prematurity/ bronchopulmonary dysplasia (BPD) are also at increased risk of being hospitalized with RSV-associated respiratory illness [5]. These infants are also more likely to be hospitalized for a longer period with respiratory infections and are more likely to require specialized care than other children [10].

\section{The Australian perspective}

There are few reports of childhood RSV-associated respiratory illness in Australia [11-14]. While these studies provide some insights into the epidemiology of RSV disease in Australia, they do not quantify the populationlevel burden of disease [12, 15]. Population-based incidence data are necessary to identify specific populations at risk of severe disease in order to prioritize limited health resources for targeted interventions and country-specific requirements for preventive strategies. Population-based estimates also allow comparison of disease burden across different countries. Furthermore, there are limited data regarding the burden of RSV disease in specific sub-populations at high risk including preterm babies and children with BPD in Australia, and no data on Indigenous children despite these children being at an elevated risk [16]. New South Wales (NSW) is the most densely populated state of the country with almost $50 \%$ of the country's Indigenous population living in the state [17].

The aim of this study was to provide updated population-based rates of RSV-associated severe childhood illness in children aged $<5$ years living in NSW. A comprehensive population-based retrospective analyses of linked administrative datasets was used to achieve this aim.

\section{METHOD}

\section{Ethics approval}

The project was approved by the NSW Population and Health Service Research Ethics Committee (HREC/09/ CIPHS/33; 2009/05/155) and the Aboriginal Health and Medical Research Council Ethics Committee (726/10).

\section{Study population and follow-up}

The study population comprised all children who were born in and resided at birth in NSW between 2001 and 2010. Each child was followed from birth until the end of the study period (31 December 2010) or until the child reached the age of 5 years or died, whichever was earlier.

\section{Data sources}

NSW Perinatal Data Collection (PDC)

The PDC records all births that are registered in NSW and contains information on mothers and babies comprising birth outcomes such as date of birth, sex of the baby, birth weight, GA and perinatal deaths of babies. The PDC also contains demographic information relating to postcode and state of residence at birth and Indigenous status of the mothers. This dataset was the primary dataset to define the birth cohort for this study.

\section{Admitted Patient Data Collection (APDC)}

The APDC contains information regarding all separations (discharges, transfers, deaths) following admission to all public and private hospitals of NSW. The data includes age at hospitalization, primary cause of hospitalization, the length of stay, transfer, discharge status, mode of separation, and the cost weight for each hospitalization. The APDC provided the information regarding all respiratory hospitalizations in the study birth cohort.

\section{Neonatal Intensive Care Units' (NICUs) data collection}

The NICUs data collection contains information on children (and their mothers) who meet the registration criteria and are admitted to one of the nine NICUs in NSW. This database was used to identify all children with $\mathrm{BPD}$ and preterm (GA $\leqslant 31$ weeks requiring admission in one of the NICUs) children in the study cohort. 


\section{Virological surveillance data}

The Eastern Sydney Laboratory Surveillance Programme conducted by the public health unit of the South Eastern Sydney Local Health District receives a weekly count of RSV detections across all age groups from three pathology laboratories with wide catchment in NSW [18]. This sentinel surveillance data is broadly representative of NSW and was used to determine the trend of RSV-associated respiratory illness transmitted in the community.

\section{Data linkage}

The Centre for Health Record Linkage $(\mathrm{CHeReL})$ is the agency responsible for linking of NSW government health databases (www.cherel.org.au) to each other for the purpose of research. $\mathrm{CHeReL}$ follows best practice and applies probabilistic linkage [19] to combine personal information in the PDC, APDC and NICUs records to produce a person-based dataset using the NSW PDC as the primary dataset to which all other datasets are linked. Each child is assigned a patient project number (PPN) and this was attached to the records in each source database. All other personal identifiers were removed from each of the datasets and the de-identified datasets with the unique identifier key were provided to the study investigators. One of the study investigators (N.H.) combined records of the same child in the three datasets using the unique identifier key and undertook data cleansing. As the PDC was the master dataset, any records for children in the APDC and NICUs datasets that did not match a PDC record were excluded from the linked data. Infants born outside of NSW were thus excluded from our birth cohort. There were 111 PDC records identified through the linkage as duplicates that were also excluded.

\section{High-risk children}

The cohort children were divided into four high-risk groups: children born to Indigenous mothers; children with BPD; preterm children without BPD; and term children born with low birthweight.

(1) Indigenous children. Children of mothers whose ethnicity was recorded as Aboriginal or Torres Strait Islander in PDC, APDC or NICUs datasets were considered to be Indigenous. This did not include Indigenous children who have an Indigenous father and a non-Indigenous mother. Indigenous children were further divided into two groups: Indigenous children with BPD, born preterm or born with low birthweight were classified as Indigenous high-risk children; all other Indigenous children were classified as Indigenous standard-risk children.

(2) Children with BPD. Non-Indigenous children who were born alive at GA $\leqslant 32$ weeks and required oxygen and/or any type of respiratory support on the day they reached 36 weeks GA were classified as children with BPD [20].

(3) Preterm children. We categorized all nonIndigenous babies born with $\mathrm{GA}<37$ weeks into three categories [21]: 32-36 weeks GA, 28-31 weeks GA and $<28$ weeks GA. None of these children were diagnosed with BPD.

(4) Term children with low birthweight. Non-Indigenous babies born at $\mathrm{GA} \geqslant 37$ weeks but with birth weight $<2500$ g.

\section{Outcome definitions}

ALRI and RSV-coded hospitalizations. The International Classification of Diseases, 10th edition (ICD-10), primary diagnostic codes associated ALRI and RSV infection were used to identify all ALRI and RSV-coded hospitalizations from APDC. Primary diagnostic codes listed as whooping cough (A37), pneumonia (J12-J18), bronchiolitis (J21), influenza (J10-J11), unspecified ALRI (J22) and bronchitis (J20) were identified as hospitalization associated with ALRIs. All primary diagnostic codes associated with RSV pneumonia (J12.1), acute RSV bronchitis (J20.5) and acute RSV bronchiolitis (J21.0) and acute bronchiolitis (J21) during the RSV season (April-September) were considered to be hospitalization associated with RSV infection. However all the bronchiolitis cases diagnosed within the RSV season were coded as RSV bronchiolitis in our dataset. Only the primary diagnostic codes were analysed as the primary codes represent the principal diagnosis for which a patient receives admitted care. Secondary codes may represent more severe conditions that occurred during the visit and thus were not included in the analyses to limit inclusion of nosocomial RSV infection. We excluded hospitalizations that occurred within 10 days of neonatal life as these hospitalizations were most likely associated with birth or perinatal complications.

\section{Cost analysis}

The NSW Cost of Care Standards 2009/2010 [22] were used to estimate the inpatient hospital costs associated RSV infection. These standards are based on outputbased activity-based hospital costs and are used for 
NSW funding formulas for hospitals. Acute admitted and newborn costs are primarily derived from the 2006/2007 NSW Hospital Cost Data Collection which captured costs on 1.2 million episodes over 83 NSW hospitals. The standards specify standard costs and cost weights based on the Australian Refined Diagnosis Related Group Version (AR-DRG) for the acute admitted hospital services. The cost weights provide a measure of resource consumption relative to a reference value representing the average inpatient hospital admission in a given year. A cost weight of 1 represents the average cost hospital admission in NSW. At discharge, each episode of acute hospital care is assigned an AR-DRG and associated cost weight which represents its relative resource consumption relative to the reference value. For example, a cost weight of 1.89 means that the admission for a particular class of patients is $89 \%$ more costly on average than the average cost.

To estimate hospital costs for RSV admissions, we assigned the weighted 'total standard NSW public hospital cost' to the cost weights assigned to each episode of acute hospital care in the APDC using the NSW Costs of Care Standards 2009/2010 [22]. The average costs for acute admitted care (including emergency department and ICU costs) was 4280 AUD for 2009/ 2010. The average cost assigned to each cost weight was adjusted to 2009/2010 AUD using the deflators specified in the NSW Costs of Care Standards 2009/ 2010 which was $4 \cdot 61 \%$ for $2003 / 2004,2002 / 2003$ and $2001 / 2002,2 \cdot 90 \%$ for $2004 / 2005,3 \cdot 20 \%$ for $2005 /$ $2006,3 \cdot 41 \%$ for $2007 / 2008,3 \cdot 55 \%$ for $2008 / 2009$ and $3 \cdot 38 \%$ for $2009 / 2010$. For hospitalizations that resulted in transfer to other facilities, the cost was equivalent to the sum of total cost incurred at each of facility.

The AR-DRG classification system accounts for the major part of the variability within a specific ARDRG. However, the hospital cost data in NSW suggest that there is an additional $10 \%$ cost typically incurred by Indigenous patients. Thus in line with the NSW Cost of Care Standards 2009/2010 [22]; the costs of episodes of care for Indigenous children were adjusted by a factor of $10 \%$ to reflect the additional care requirements and resource consumption [22]. We divided the total cost of all RSV-coded hospitalizations identified over the 10-year period by 10 to estimate the mean annual cost of RSV-coded hospitalizations.

\section{Statistical analysis}

We used descriptive statistics to describe the profile of the cohort. Using the dates of respiratory hospitalization from the APDC and the laboratory detection of RSV infections from the virological surveillance data we plotted week and year specific timing of ARI and RSV hospitalizations and RSV circulation in NSW for the study period.

We calculated incidence as the number of new events divided by child-years at risk. A lag of 14 days between two successive respiratory hospitalizations was considered to be a new episode of respiratory hospitalization. Two episodes of respiratory hospitalizations within 48 $\mathrm{h}$ and with the same primary ICD-10 AM code were considered continuation of the same illness episode. Moreover, an episode of hospitalization for which the child was transferred to another hospital was considered as continuation of the same illness episode. Children were not considered to be at risk of RSV hospitalization while in the hospital. Person time at risk was calculated by subtracting the number of days spent in hospital from the number of days followed up. Children were divided in to five age groups (0-3 months, 4-6 months, 7-11 months, 12-24 months, $2-<5$ years). We used Poisson estimation to calculate 95\% confidence intervals (CIs) around incidences. All analyses were performed using Stata release 13 (StataCorp LP, USA).

\section{RESULTS}

\section{Profile of the cohort}

During 2001-2010, we followed 870314 children for 3267790 person-years ( 3.8 years/child) and $2295(0.3 \%)$ children died while hospitalized during the follow-up period. A total of $94082(11 \%)$ children belonged to one of the high-risk groups (Table 1).

\section{Seasonality of RSV illness}

Weekly counts of ALRI hospitalization, RSV-coded hospitalization and RSV detection followed similar patterns in NSW over the 10-year period. There was distinct seasonality of ALRI and RSV hospitalizations in NSW. The peak in RSV-coded hospitalizations was between May and August of each year with $81 \%$ of the total RSV-coded hospitalizations recorded between these months (Fig. 1).

\section{Incidence of ALRI and RSV hospitalizations}

Over the 10-year period there were 68913 new episodes of ALRI hospitalization. The incidence of ALRI hospitalization (per 1000 child-years) was 
Table 1. Descriptive profile of the cohort children aged $<5$ years born in NSW between 2001 and 2010

\begin{tabular}{ll}
\hline \hline Characteristics & Frequency \\
\hline Total number of children followed & 870314 \\
Total person time (years) & $3261790(3 \cdot 8$ years/child) \\
Male sex of the child (\%) & $447942(51)$ \\
Median birthweight, g (IQR) & $3410(\mathrm{IQR} 3070-3745)$ \\
Median GA, weeks (IQR) & $39(38-40)$ \\
Indigenous children (\%) & $28486(3)$ \\
High-risk children & $3939(14)$ \\
Healthy children & $24547(86)$ \\
Children with BPD (\%) & $823(0 \cdot 1)$ \\
Preterm children (\%) & \\
GA 32-36 weeks & $45213(5)$ \\
GA 28-31 weeks & $3779(0 \cdot 4)$ \\
GA<28 weeks & $1380(0 \cdot 2)$ \\
Children born at term with low birthweight (\%) & $15224(2)$ \\
All-cause associated acute lower respiratory hospitalizations & \\
Total number of new episodes & 68913 \\
Median age at hospitalization, months (IQR) & $9(4-17)$ \\
Median length of hospitalization, days (IQR) & $2(1-3)$ \\
RSV-coded hospitalizations & \\
Total number of new episodes & 16119 \\
Median age at hospitalization, months (IQR) & $(23 \%$ of all respiratory hospitalization) \\
Male sex of the child (\%) & $5(2-9)$ \\
Median length of hospitalization, days (IQR) & $9210(57)$ \\
ICD-10 AM coding of RSV-coded hospitalizations & $3(2-4)$ \\
RSV bronchiolitis (\%) & \\
RSV pneumonia (\%) & $15393(95 \cdot 5)$ \\
RSV bronchitis (\%) & $643(4)$ \\
\hline \hline
\end{tabular}

BPD, Bronchopulmonary dysplasia; GA, gestational age; IQR, interquartile range; RSV, respiratory syncytial virus.

$21 \cdot 1(95 \%$ CI $21 \cdot 0-21 \cdot 3)$. The incidence (per 1000 child-years) of ALRI hospitalization was greater $(24 \cdot 5,95 \%$ CI $24 \cdot 2-24 \cdot 7)$ in male children than female children (17·7, 95\% CI 17.5-17.9). The incidences (per 1000 child-years) of ALRI hospitalization for children aged 0-3 months, 4-6 months, 7-11 months, 12-24 months and $2-<5$ years, respectively, were $69 \cdot 9(95 \%$ CI $67 \cdot 8-70 \cdot 0), 64 \cdot 0(95 \%$ CI $63 \cdot 0-65 \cdot 2), 25 \cdot 8(95 \%$ CI $24 \cdot 8-25 \cdot 6), 21 \cdot 7(95 \%$ CI $21 \cdot 3-22 \cdot 0)$ and $7 \cdot 0$ $(95 \%$ CI $6 \cdot 8-7 \cdot 1)$.

Of 68913 new episodes of ALRI hospitalization, 16 $119(23 \%)$ were coded as RSV (Table 1$)$. In this birth cohort, $35 \cdot 5 \%$ (5720/16 119) of all RSV hospitalizations occurred in children aged 0-3 months, $24 \cdot 0 \%(3836 / 16119)$ in children aged 4-6 months, $24 \cdot 0 \%(3809 / 16119)$ in children aged 7-11 months, $13 \cdot 0 \%$ (2133/16 119) in children aged $12-24$ months and $3 \cdot 0 \%(518 / 16119)$ in children aged $2-<5$ years.

The population-based incidence (per 1000 childyears) of RSV hospitalization for children aged $<5$ years was $4.9(95 \%$ CI $4 \cdot 8-5 \cdot 0)$; the incidence was 5.6
(95\% CI $5 \cdot 5-5 \cdot 7)$ for boys and $4 \cdot 3(95 \%$ CI $4 \cdot 2-4 \cdot 4)$ for girls. The incidence of RSV hospitalization (per 1000 child-years) was 11.0 for Indigenous children, 20.9 (95\% CI 18.6-23.4) for Indigenous high-risk children and $9 \cdot 1(95 \%$ CI 8.5-9.7) for Indigenous standard-risk children, 81.5 (95\% CI 71.0-93.5) for children with BPD, $10 \cdot 2(95 \%$ CI $9 \cdot 7-10 \cdot 7)$ for preterm children with GA 32-36 weeks, $27 \cdot 0(95 \%$ CI $23 \cdot 9-30 \cdot 3)$ for preterm children with GA $28-31$ weeks, $39 \cdot 0$ (95\% CI $31 \cdot 0$ 49.0) for preterm children with GA $<28$ weeks and $6 \cdot 7$ (95\% CI 6 5-7.4) for term children with low birthweight. The age-specific rate of RSV hospitalizations was highest in children with BPD (Table 2).

\section{Cost of illness}

The mean cost of each episode of RSV hospitalization in children aged <5 was AUD 6350 (95\% CI 6157-6543). For Indigenous children the mean cost for each episode of RSV hospitalization was AUD 9190 (95\% CI 801810273 ). The mean cost associated with each episode of 


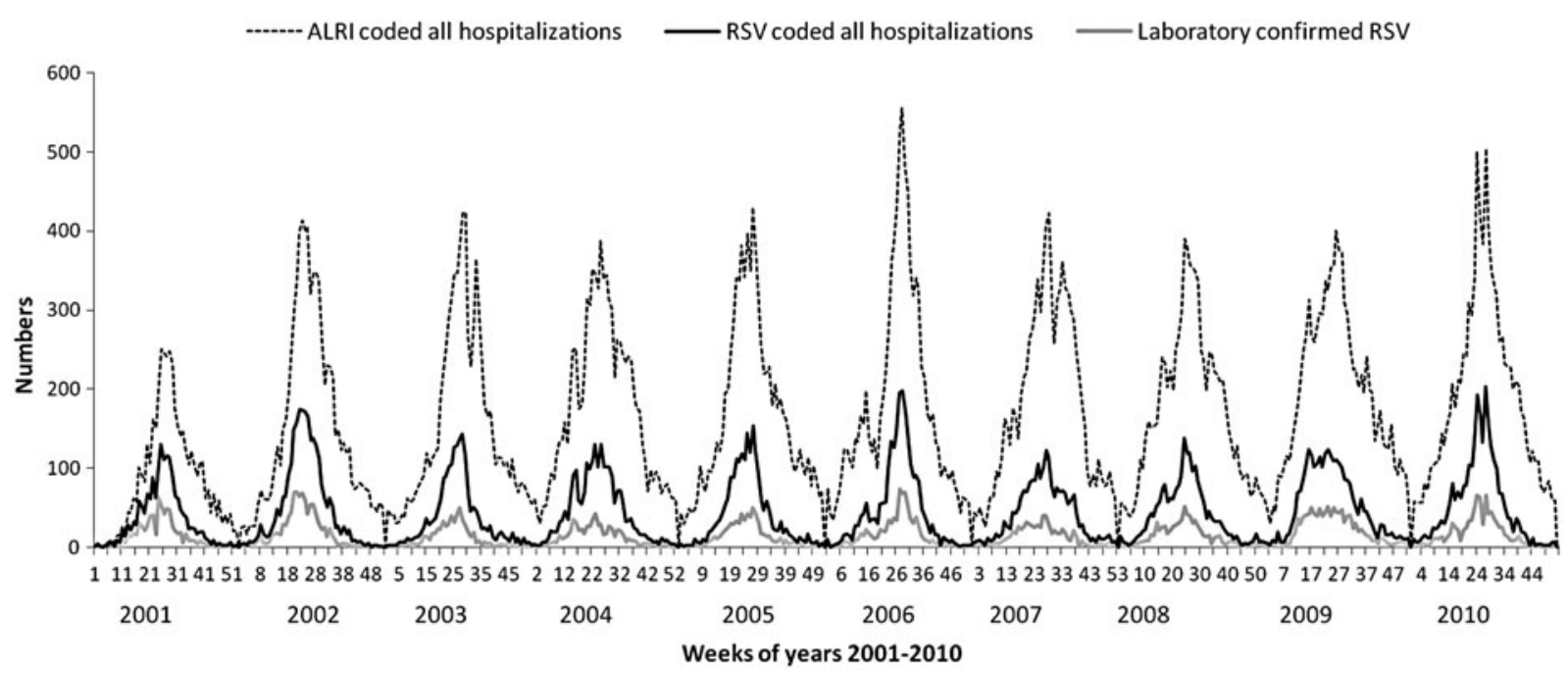

Fig. 1. Seasonality of acute lower respiratory tract infections (ALRIs) and respiratory syncytial virus (RSV)-coded hospitalizations in children aged $<5$ years, NSW, 2001-2010.

hospitalization for children with BPD, preterm children and children born with low birthweight were AUD 12 731 (95\% CI 9212- 16251), AUD 9354 (95\% CI $8477-$ 10233 ) and AUD 6664 (95\% CI 5523-7805), respectively. The mean cost for all other term children was AUD 5649 (95\% CI 5475-5823). The mean annual inpatient hospital cost associated with RSV hospitalization in NSW was AUD 9080000.

\section{DISCUSSION}

This is the first study to provide a comprehensive population-based burden of severe childhood RSV illness in Australian children including in Indigenous children. The burden of hospitalization was exceptionally high in Indigenous children aged $<2$ years; more than double the rates in the contemporaneous non-Indigenous population. The estimated rates in Australian Indigenous children were comparable to estimates from American Native children [9, 23]. Respiratory infection has been identified as one of the major causes for hospitalizations in Indigenous children [16]. This disproportionate burden of disease in Indigenous children confirms that this population continues to be at risk of poorer health outcomes compared to other children [24]. Such high burden of disease in Indigenous children has been attributed to poor access to healthcare services, household crowding, lack of sanitation facilities and indoor exposure to high levels of smoke [9]. The Indigenous population of Australia continue to also suffer from social inequalities [17] highlighting that improving Indigenous health will require setting priorities for improving socioeconomic indicators of the community.

Our population-based incidence of $4 \cdot 9 / 1000$ childyears for RSV hospitalization in children aged $<5$ years was more than the population-based estimates of $3-3 \cdot 5 / 1000$ children in the same age group in the United States [6, 25]. The incidence was highest in children aged $<6$ months, specifically in those aged $<3$ months. Although admission criteria and case definitions may vary from country to country, other studies have also demonstrated that the burden remains highest in children aged $<6$ months [25-27]. Severe RSV disease in very young children is associated with chronic morbidity including recurrent wheezing and asthma [28, 29]. In addition, the average cost of AUD 6350 per RSV-associated hospitalization was higher than average cost of AUD 5163 per episode of paediatric hospitalization for acute care in NSW [30]. The cost was also higher than the mean cost of US\$3799 (equivalent to AUD 5303) associated with each childhood bronchiolitis hospitalization in the United States [31]. These factors make development of a RSV vaccine a public health priority in Australia. Although there are several RSV vaccines and novel anti-RSV therapies in different phases of clinical trials [32-34], an effective therapy or vaccine is yet to be licensed. When a vaccine becomes available, a potential strategy for vaccination may be passive immunity through maternal immunization for children aged $<6$ months and active immunization for children aged 6-12 months [35]. 
Table 2. Incidences of RSV hospitalization in children aged $<5$ years in NSW, 2001-2010

\begin{tabular}{|c|c|c|c|}
\hline Cohort & $\begin{array}{l}\text { Number of RSV-coded } \\
\text { hospitalizations }\end{array}$ & Person-years & IR/1000 child-years $(95 \% \mathrm{CI})$ \\
\hline \multicolumn{4}{|l|}{ 0-3 months } \\
\hline Indigenous children & 392 & 6793 & $58 \cdot 0(49 \cdot 5-60 \cdot 5)$ \\
\hline High-risk children & 105 & 833 & $126 \cdot 0(103 \cdot 0-152 \cdot 5)$ \\
\hline Healthy children & 287 & 5960 & $48 \cdot 0(42 \cdot 7-54 \cdot 0)$ \\
\hline Children with BPD & 3 & 13 & $239 \cdot 0(47 \cdot 5-674 \cdot 4)$ \\
\hline Children with GA 32-36 weeks & 539 & 9200 & $58 \cdot 5(53 \cdot 7-63 \cdot 7)$ \\
\hline Children with GA 29-31 weeks & 62 & 1052 & $173(133-75 \cdot 5)$ \\
\hline Children with $\mathrm{GA} \leqslant 28$ weeks & 0 & 30 & 0 \\
\hline Term children with low birthweight & 145 & 3402 & $42 \cdot 6(36 \cdot 0-50 \cdot 0)$ \\
\hline All other term healthy children & 4579 & 178525 & $25 \cdot 6(23 \cdot 9-25 \cdot 3)$ \\
\hline \multicolumn{4}{|l|}{ 4-6 months } \\
\hline Indigenous children & 348 & 6478 & $53 \cdot 7(48 \cdot 2-59 \cdot 6)$ \\
\hline High-risk children & 80 & 837 & $95 \cdot 5(75 \cdot 7-119 \cdot 0)$ \\
\hline Healthy children & 268 & 5640 & $47 \cdot 5(42 \cdot 0-53 \cdot 5)$ \\
\hline Children with BPD & 38 & 138 & $275 \cdot 0(194 \cdot 8-378 \cdot 0)$ \\
\hline Children with GA 32-36 weeks & 367 & 9106 & $40 \cdot 0(36 \cdot 2-44 \cdot 6)$ \\
\hline Children with GA 28-31 weeks & 89 & 638 & $139 \cdot 4(112 \cdot 0-171 \cdot 6)$ \\
\hline Children with $\mathrm{GA}<28$ weeks & 27 & 132 & $205 \cdot 0(134 \cdot 7-297 \cdot 6)$ \\
\hline Term children with low birthweight & 92 & 3275 & $28 \cdot 0(22 \cdot 6-34 \cdot 5)$ \\
\hline All other term healthy children & 2869 & 171202 & $16 \cdot 7(16 \cdot 2-17 \cdot 4)$ \\
\hline \multicolumn{4}{|l|}{ 7-11 months } \\
\hline Indigenous children & 268 & 27178 & $9 \cdot 8(8 \cdot 7-11 \cdot 1)$ \\
\hline High-risk children & 69 & 3856 & $17 \cdot 8(14 \cdot 0-22 \cdot 6)$ \\
\hline Healthy children & 199 & 23322 & $8 \cdot 5(7 \cdot 4-9 \cdot 8)$ \\
\hline Children with BPD & 71 & 733 & $96 \cdot 8(75 \cdot 6-122 \cdot 2)$ \\
\hline Children with GA 32-36 weeks & 378 & 36663 & $10 \cdot 3(9 \cdot 3-11 \cdot 4)$ \\
\hline Children with GA 28-31 weeks & 88 & 3,269 & $27 \cdot 0(21 \cdot 5-33 \cdot 0)$ \\
\hline Children with $\mathrm{GA}<28$ weeks & 34 & 631 & $53 \cdot 8(37 \cdot 3-75 \cdot 2)$ \\
\hline Term children with low birthweight & 79 & 12253 & $6 \cdot 4(5 \cdot 0-8 \cdot 0)$ \\
\hline All other term healthy children & 289 & 617877 & $4 \cdot 7(4 \cdot 5-4 \cdot 8)$ \\
\hline \multicolumn{4}{|l|}{ 12-24 months } \\
\hline Indigenous children & 114 & 22288 & $5 \cdot 0(4 \cdot 2-6 \cdot 0)$ \\
\hline High-risk children & 38 & 3036 & $12 \cdot 5(8 \cdot 8-17 \cdot 2)$ \\
\hline Healthy children & 76 & 19251 & $4 \cdot 0(3 \cdot 0-5 \cdot 0)$ \\
\hline Children with BPD & 69 & 712 & $96 \cdot 8(75 \cdot 4-122 \cdot 6)$ \\
\hline Children with GA 32-36 weeks & 210 & 31302 & $6 \cdot 7(5 \cdot 8-7 \cdot 7)$ \\
\hline Children with GA 28-31 weeks & 31 & 2414 & $12 \cdot 8(8 \cdot 7-18 \cdot 2)$ \\
\hline Children with $\mathrm{GA}<28$ weeks & 14 & 471 & $29 \cdot 7(16 \cdot 2-49 \cdot 8)$ \\
\hline Term children with low birthweight & 52 & 11403 & $4 \cdot 5(3 \cdot 4-5 \cdot 0)$ \\
\hline All other term healthy children & 1643 & 583872 & $2 \cdot 8(2 \cdot 7-2 \cdot 9)$ \\
\hline \multicolumn{4}{|l|}{$2-<5$ years } \\
\hline Indigenous children & 16 & 44603 & $0 \cdot 35(0 \cdot 2-0 \cdot 6)$ \\
\hline High-risk children & 4 & 5614 & $0 \cdot 7(0 \cdot 02-2 \cdot 0)$ \\
\hline Healthy children & 12 & 38988 & $0 \cdot 3(0 \cdot 2-0 \cdot 5)$ \\
\hline Children with BPD & 21 & 893 & $23 \cdot 5(14 \cdot 5-36 \cdot 0)$ \\
\hline Children with GA 32-36 weeks & 54 & 66076 & $0 \cdot 8(0 \cdot 6-1 \cdot 0)$ \\
\hline Children with GA 28-31 weeks & 17 & 3986 & $4 \cdot 2(0 \cdot 8-12 \cdot 0)$ \\
\hline Children with $\mathrm{GA}<28$ weeks & 3 & 719 & $0 \cdot 5(0 \cdot 01-1)$ \\
\hline Term children with low birthweight & 10 & 41299 & $0 \cdot 4(0 \cdot 2-0 \cdot 7)$ \\
\hline All other term healthy children & 385 & 1378996 & $0 \cdot 27(0 \cdot 25-0 \cdot 31)$ \\
\hline
\end{tabular}

BPD, Bronchopulmonary dysplasia; CI, confidence interval; GA, gestational age, IR, incidence rate; RSV, respiratory syncytial virus. 
Similar to other studies, our findings also suggest that RSV-associated hospitalization was highest for children with BPD [36, 37]. While rates of hospitalization for other children at high risk decreased beyond age 2 years, it continued to be high for children with BPD. Preterm children in this cohort also had very high rates of hospitalization. Palivizumab, a monoclonal antibody, can significantly reduce hospitalizations in high-risk infants $[38,39]$. While many developed countries use palivizumab for preventing RSV hospitalization in high-risk target groups and have demonstrated its effectiveness [38, 39], prophylactic use of palivizumab remains limited in Australia. In addition there is no standard guideline for its administration. Further well-designed research evaluating the effectiveness and cost-effectiveness of palivizumab in highrisk children especially the Indigenous or preterm infants with or without BPD, may help standardize policy guidelines for lowering the disease burden.

Our study has several limitations. First, we had the data linked until 2010. Linkage of multiple datasets is a time-intensive process and this was the most updated linked data we had access to. There has been no change in treatment of RSV respiratory disease in Australia and our data suggest that number of RSV-coded hospitalizations has remained more or less constant over the 10-year period. Our study has demonstrated that publicly available linked administrative data can be effectively used to quantify disease burden in a population. There may have been missing or incorrect links which is inherent to studies based on large linked datasets. However, $\mathrm{CHeReL}$ routinely performs sensitivity analysis for the quality control of the linkage done by them. For our particular study the false-positive rate, i.e. a child in PDC being wrongly linked to an observation in any other dataset was $0 \cdot 3 \%$. The estimated rates were based on RSV-coded hospitalization which included confirmed and probable RSV hospitalizations and may have resulted in misclassification as testing for RSV is not routine in the clinical setting in NSW. All the RSV-coded hospitalizations were recorded within the RSV season and the RSV-coded hospitalizations and laboratory-confirmed RSV detection rate followed a similar trend suggesting the validity of our estimates. Although RSV is known to be associated with asthma exacerbation and wheeze in children [28], we did not include such hospitalizations. This may have led to underestimation of the burden of RSV; however, we were interested in hospitalizations where RSV was the primary diagnosis for admitted care. We only estimated the rates of RSV hospitalization and there may be significant burden on healthcare services associated with mild RSV illness. However, the aim of the study was to estimate the burden of severe RSV illness so that that data can inform policy decisions regarding prioritizing RSV-specific interventions for specific groups of high-risk children. Although RSV-associated deaths are infrequent [40], we did not have access to the cause-of-death database which limited estimation of RSV-associated mortality rates in this cohort.

The study findings have several important policy implications. The findings confirm that RSV is a major cause of childhood hospitalization, particularly for children aged $<3$ months in Australia. The burden continues to be highest for Indigenous children and children with BPD and born preterm. However, the high-risk children only accounted for $11 \%$ of the study cohort and $23 \%$ of all RSV hospitalizations. Most of the disease burden was in otherwise healthy children highlighting the unmet need for an effective vaccine or therapy.

\section{ACKNOWLEDGEMENTS}

The authors are grateful to Respiratory Department of Sydney Children's Hospital, Randwick and Virology Research Laboratory of Prince of Wales Hospital, Randwick for their cooperation. The authors thank the Directors, the NICUs members and the audit officers of all tertiary units in supporting this collaborative study: NICUc, Dr Jennifer Bowen (Chairperson), Barbara Bajuk (Coordinator), Sara Sedgley (Research Officer); Canberra Hospital, Dr Hazel Carlisle (Director), Prof. Alison Kent, Judith Smith; John Hunter Children's Hospital, Dr Paul Craven (Director), Lynne Cruden, Alissa Argomand; Royal Prince Alfred Hospital, Ingrid Rieger (Director), Dr Girvan Malcolm, Tracey Lutz (Clinical Director), Shelley Reid; Liverpool Hospital, Dr Jacqueline Stack (Director), Dr Ian Callander, Kathryn Medlin, Kaye Marcin; Nepean Hospital, Dr Vijay Shingde, Mee Fong Chin, Kerrie Bonzer; The Children's Hospital at Westmead, Prof. Nadia Badawi (Director), Dr Robert Halliday, Caroline Karskens; Royal North Shore Hospital, Dr Mary Paradisis (Director), A/Prof. Martin Kluckow, Claire Jacobs; Sydney Children's Hospital, Dr Andrew Numa (Director), Dr Gary Williams, Janelle Young; Westmead Hospital, Dr Melissa Luig (Director), Jane Baird; and Royal Hospital for Women, A/Prof. 
Kei Lui (Director), Dr Ju-Lee Oei, Diane Cameron. We also thank the babies and their families, the nursing and midwifery, obstetric and medical records staff of the obstetric and children's hospitals in NSW and the ACT.

\section{DECLARATION OF INTEREST}

None.

\section{REFERENCES}

1. Leader S, Kohlhase K. Respiratory syncytial virus-coded pediatric hospitalizations, 1997 to 1999. Pediatric Infectious Disease Journal 2002; 21: 629-632.

2. Hall CB. Respiratory syncytial virus and parainfluenza virus. New England Journal of Medicine 2001; 344: 1917-28.

3. Yun BY, et al. Viral etiology and epidemiology of acute lower respiratory tract infections in Korean children. Pediatric Infectious Disease Journal 1995; 14: 1054 1059.

4. Weissenbacher M, et al. Etiologic and clinical evaluation of acute lower respiratory tract infections in young Argentinian children: an overview. Reviews of Infectious Diseases 1990; 12 (Suppl. 8): S889-98.

5. Simoes EA. Respiratory syncytial virus infection. Lancet 1999; 354: 847-852.

6. Iwane MK, et al. Population-based surveillance for hospitalizations associated with respiratory syncytial virus, influenza virus, and parainfluenza viruses among young children. Pediatrics 2004; 113: 1758-1764.

7. Shay DK, et al. Bronchiolitis-associated hospitalizations among US children, 1980-1996. Journal of the American Medical Association 1999; 282: 1440-1446.

8. Holman RC, et al. Infectious disease hospitalizations among American Indian and Alaska native infants. Pediatrics 2003; 111: E176-182.

9. Holman RC, et al. Respiratory syncytial virus hospitalizations among American Indian and Alaska Native infants and the general United States infant population. Pediatrics 2004; 114: e437-444.

10. Madhi SA, et al. Respiratory syncytial virus associated illness in high-risk children and national characterisation of the circulating virus genotype in South Africa. Journal of Clinical Virology 2003; 27: 180-189.

11. Dede A, et al. Respiratory syncytial virus infections in Central Australia. Journal of Paediatrics and Child Health 2010; 46: 35-39.

12. Ranmuthugala G, Brown L, Lidbury BA. Respiratory syncytial virus - the unrecognised cause of health and economic burden among young children in Australia. Communicable Disease Intelligence Quarterly Report 2011; 35: 177-184.

13. Lister S, Mcintyre P, Menzies R. The epidemiology of respiratory syncytial virus infections in NSW children, 1992-1997. New South Wales Public Health Bulletin 2000; 11: 119-123.
14. De Silva LM, Hanlon MG. Respiratory syncytial virus: a report of a 5-year study at a children's hospital. Journal of Medical Virology 1986; 19: 299-305.

15. Roche $\mathbf{P}$, Lambert S, Spencer J. Surveillance of viral pathogens in Australia: respiratory syncytial virus. Communicable Disease Intelligence Quarterly Report 2003; 27: 117-122.

16. Torzillo PJ, Chang AB. Acute respiratory infections among Indigenous children. Medical Journal of Australia 2014; 200: 559-560.

17. Australian Government. The health and welfare of Australia's Aboriginal and Torres Strait Islander people an overview. Australian Institute of Health and Welfare, 2011.

18. Schindeler SK, et al. Evaluation of alternative respiratory syndromes for specific syndromic surveillance of influenza and respiratory syncytial virus: a time series analysis. BMC Infectious Diseases 2009; 9: 190 .

19. Irvine KA, Taylor LK. The Centre for Health Record Linkage: fostering population health research in NSW. NSW Public Health Bulletin 2011; 22: 17-18.

20. Bancalari E, Claure N, Sosenko IR. Bronchopulmonary dysplasia:changes in pathogenesis, epidemiology and definition. Seminars in Neonatology 2003; 8: 63-71.

21. Moutquin JM. Classification and heterogeneity of preterm birth. An International Journal of Obstetrics \& Gynaecology 2003; 110 (Suppl. 20): 30-33.

22. NSW Government Ministry of Health. Costs of Care Standards 2009/10. 2011.

23. Karron RA, et al. Severe respiratory syncytial virus disease in Alaska native children. RSV Alaska Study Group. Journal of Infectious Diseases 1999; 180: 41-49.

24. Vos T, et al. Burden of disease and injury in Aboriginal and Torres Strait Islander Peoples: the Indigenous health gap. International Journal of Epidemiology 2009; 38: 470-477.

25. Hall CB, et al. The burden of respiratory syncytial virus infection in young children. New England Journal of Medicine 2009; 360: 588-598.

26. Khuri-Bulos N, et al. Burden of respiratory syncytial virus in hospitalized infants and young children in Amman, Jordan. Scandinavian Journal of Infectious Diseases 2010; 42: 368-374.

27. Nokes DJ, et al. Respiratory syncytial virus infection and disease in infants and young children observed from birth in Kilifi District, Kenya. Clinical Infectious Diseases 2008; 46: 50-57.

28. Henderson $\mathbf{J}$, et al. Hospitalization for RSV bronchiolitis before 12 months of age and subsequent asthma, atopy and wheeze: a longitudinal birth cohort study. Pediatric Allergy and Immunology 2005; 16: 386-392.

29. Sigurs N, et al. Respiratory syncytial virus bronchiolitis in infancy is an important risk factor for asthma and allergy at age 7. American Journal of Respiratory and Critical Care Medicine 2000; 161: 1501-1507.

30. NSW Government. NSW Health Activity Based Management Portal. 2013-2014. 
31. Pelletier AJ, Mansbach JM, Camargo Jr. CA. Direct medical costs of bronchiolitis hospitalizations in the United States. Pediatrics 2006; 118: 2418-2423.

32. Resch B. Burden of respiratory syncytial virus infection in young children. World Journal of Clinical Pediatrics 2012; 1: 8-12.

33. Mackman RL, et al. Discovery of an oral respiratory syncytial virus (RSV) fusion inhibitor (GS-5806) and clinical proof of concept in a human RSV challenge study. Journal of Medicinal Chemistry 2015; 58: 1630-1643.

34. DeVincenzo JP, et al. Oral GS-5806 activity in a respiratory syncytial virus challenge study. New England Journal of Medicine 2014; 371: 711-722.

35. Iwane MK, et al. Population-based surveillance for hospitalizations associated with respiratory syncytial virus, influenza virus, and parainfluenza viruses among young children. Pediatrics 2004; 113: 1758-1764.
36. Boyce TG, et al. Rates of hospitalization for respiratory syncytial virus infection among children in medicaid. Journal of Pediatrics 2000; 137: 865-870.

37. Groothuis JR, Gutierrez KM, Lauer BA. Respiratory syncytial virus infection in children with bronchopulmonary dysplasia. Pediatrics 1988; 82: 199-203.

38. Parnes C, et al. Palivizumab prophylaxis of respiratory syncytial virus disease in 2000-2001: results from The Palivizumab Outcomes Registry. Pediatric Pulmonology 2003; 35: 484-489.

39. Romero JR. Palivizumab prophylaxis of respiratory syncytial virus disease from 1998 to 2002: results from four years of palivizumab usage. Pediatric Infectious Disease Journal 2003; 22: S46-54.

40. Byington CL, et al. Respiratory syncytial virusassociated mortality in hospitalized infants and young children. Pediatrics 2015; 135: e24-31. 